\title{
Pressure Dependence of Magnetic Properties in Fe-Mn-B amorphous alloys: Evidence for Inhomogeneous Ferromagnetism
}

L F Kiss ${ }^{1,4}$, T Kemény ${ }^{1}$, J. Bednarčík ${ }^{2}$, J Kamarád ${ }^{3}$, Z Arnold $^{3}$, Z Konôpková ${ }^{2}$ and H-P Liermann $^{2}$

\author{
${ }^{1}$ Institute for Solid State Physics and Optics, Wigner Research Centre for Physics, HAS, H- \\ 1525 Budapest, P.O.Box 49, Hungary \\ ${ }^{2}$ Deutsches Elektronen Synchrotron DESY, Notkestrasse 85, D-22603 Hamburg, Germany \\ ${ }^{3}$ Institute of Physics AS CR, v.v.i., Na Slovance 2, 18221 Praha 8, Czech Republic
}

E-mail: kissl@szfki.hu

PACS numbers: 75.50.Kj, 62.50.-p, 75.30.Kz, 75.30.Cr

\begin{abstract}
The pressure dependence of the saturation magnetization and Curie temperature was studied in melt-spun $\mathrm{Fe}_{60} \mathrm{Mn}_{20} \mathrm{~B}_{20}, \mathrm{Fe}_{56} \mathrm{Mn}_{24} \mathrm{~B}_{20}$ and $\mathrm{Fe}_{75} \mathrm{~B}_{25}$ amorphous alloys up to 0.9 GPa corresponding to volume changes up to $0.45 \%$. In addition, in-situ high-pressure (up to $40 \mathrm{GPa}) \mathrm{X}$-ray diffraction was performed to determine the compressibility of the latter two alloys. Both the Curie temperature $T_{\mathrm{C}}$ (at atmospheric pressure $T_{\mathrm{C}}=201 \pm 3$ and $159 \pm 3 \mathrm{~K}$ ) and the low-temperature saturation magnetization $M_{5 \mathrm{~K}, 5 \mathrm{~T}}$ decrease remarkably with increasing pressure: $\mathrm{d} T_{\mathrm{C}} / \mathrm{d} p=-31 \pm 0.5$ and $-32 \pm 5 \mathrm{~K} / \mathrm{GPa}$ and $\mathrm{d} \ln M_{5 \mathrm{~K}, 5 \mathrm{~T}} / \mathrm{d} p=-0.15 \pm 0.02$ and $-0.13 \pm$ $0.03 \mathrm{GPa}^{-1}$ for $x_{\mathrm{Mn}}=20$ and 24 at $\%$, respectively. Compared to $\mathrm{d} \ln M_{5 \mathrm{~K}, 5 \mathrm{~T}} / \mathrm{d} p=-0.016 \pm 0.003$
\end{abstract}


$\mathrm{GPa}^{-1}$ measured for $\mathrm{Fe}_{75} \mathrm{~B}_{25}$, the pressure dependence of $M_{5 \mathrm{~K}}$, 5 is one order of magnitude larger in the ternary alloys. The bulk moduli for the $\mathrm{Fe}_{56} \mathrm{Mn}_{24} \mathrm{~B}_{20}$ and $\mathrm{Fe}_{75} \mathrm{~B}_{25}$ glasses were measured to be $152 \mathrm{GPa}$ and $173 \mathrm{GPa}$, respectively. These data are also compared with the pressure dependence of the hyperfine-field and theoretical calculations of the saturation moment for Fe-B alloys reported in the literature. The results were interpreted within an inhomogeneous itinerant-electron model of ferromagnetism.

4 To whom any correspondence should be addressed. 


\section{INTRODUCTION}

The pressure dependent magnetoelastic properties of Fe-based amorphous alloys are of high scientific interest for at least two reasons. Generally, they shed light on the fundamental aspects of the relation between the volume stability (i.e. volume dependence) of the Fe moment and the features of the electronic structure of Fe-based materials. Besides that, recently, the Fe-based amorphous alloys have been used as starting materials for the preparation of useful nanostructures. Pressure changes the average atomic volume and consequently influences the intrinsic magnetic properties of these alloys, e.g. Curie temperature $\left(T_{\mathrm{C}}\right)$ and saturation magnetic moment [1]. Previous studies of the pressure dependence of these quantities have been mainly focused on Fe-based amorphous alloys with Curie points close to the ambient temperature with respect to their possible application potential. The Curie points of Fe-B amorphous alloys can be significantly lowered by early transition-metal (TM) additions, e.g. Cr [2], Mo [3], Mn [4]. The pressure dependence of the Curie point for the amorphous $\mathrm{Fe}_{80-\mathrm{x}} \mathrm{Mn}_{\mathrm{x}} \mathrm{B}_{20}$ alloy system $(x=6-20$ at $\%$ ) has been investigated by Schiller et al. [5]. However, only one report is known on the pressure variation of the saturation moment for amorphous alloys [6], Fe-Ni-P-B/(-Si) alloy system). Besides this early paper, the pressure dependence of the hyperfine field determined by Mössbauer measurements for some amorphous alloys [7-9] was used as a relevant quantity for comparison with the pressure dependence of saturation magnetization. The relation between the Fe atomic magnetic moment and hyperfine field for transition metal-metalloid type metallic glasses was established in [10] and revised recently [11]. 
In the present work, a significantly larger effect of pressure on the saturation magnetization and the Curie temperature for the amorphous $\mathrm{Fe}_{56} \mathrm{Mn}_{24} \mathrm{~B}_{20}$ and $\mathrm{Fe}_{60} \mathrm{Mn}_{20} \mathrm{~B}_{20}$ alloys has been observed when compared with the binary $\mathrm{Fe}_{75} \mathrm{~B}_{25}$ amorphous alloy. For the amorphous $\mathrm{Fe}$ Mn-B alloys, no data on magnetization under high pressure have been previously known. To compare experimental and theoretical results, it is necessary to know the volume changes under pressure, therefore the compressibility of $\mathrm{Fe}_{56} \mathrm{Mn}_{24} \mathrm{~B}_{20}$ and $\mathrm{Fe}_{75} \mathrm{~B}_{25}$ was directly measured by in-situ high-pressure X-ray diffraction.

The paper is organized as follows. Section 2 gives the details of the sample preparation and characterization. It also describes the magnetic measurements, the pressure cell and the experimental setup for the compressibility studies. Sections 3.1. and 3.2. contain the experimental results of the pressure-dependent magnetic measurements and of the compressibility studies, respectively. Section 4.1. gives evidence of the magnetically inhomogeneous nature of the investigated amorphous alloys through an Arrott-plot analysis. In Sec. 4.2., the experimental results are interpreted within an inhomogeneous itinerantelectron model of ferromagnetism. Section 5 summarizes briefly the conclusions derived from the present study.

\section{EXPERIMENTAL}

\subsection{Sample preparation and characterization}

Amorphous $\mathrm{Fe}_{80-\mathrm{x}} \mathrm{Mn}_{\mathrm{x}} \mathrm{B}_{20}$ alloys with nominal values of $x=20$ and 24 at $\%$ were prepared by melt spinning in vacuum. The Mn content of the samples was $x=19.5 \pm 1$ and $22 \pm 1$ at $\%$ as measured by electron microprobe analysis and assuming the nominal B concentration. The 
nominal composition of the binary Fe-B sample was $\mathrm{Fe}_{77} \mathrm{~B}_{23}$. Although the nominal composition of the binary Fe-B sample was $\mathrm{Fe}_{77} \mathrm{~B}_{23}$, careful Mössbauer spectroscopy studies could not identify any measurable bcc-Fe fraction beyond the bct $\mathrm{Fe}_{3} \mathrm{~B}$ intermetallic compound in the crystallized ribbon. Therefore, we identify this composition as $\mathrm{Fe}_{75} \mathrm{~B}_{25}$ throughout this paper. The amorphous nature of the ribbons (with a cross section of $1 \times 0.013$ $\mathrm{mm}^{2}$ ) was verified by X-ray diffractometry, Mössbauer spectroscopy and differential scanning calorimetry (Perkin-Elmer DSC).

\subsection{Magnetic and structural studies}

The magnetic properties were investigated using the MPMS-5S superconducting quantum interference device (SQUID) magnetometer up to $5 \mathrm{~T}(50 \mathrm{kOe})$ in the temperature range of 5 $\mathrm{K} \leq T \leq 300 \mathrm{~K}$. Samples were compressed under hydrostatic pressure up to $1.2 \mathrm{GPa}$ in a miniature piston-cylinder $\mathrm{CuBe}$ pressure cell [12] using a mineral oil as the pressure transmitting medium and the cell with the sample was put into the SQUID magnetometer.

In-situ high-pressure X-ray diffraction experiments were performed at the Extreme Condition Beamline P02.2 of PETRA III (Hamburg, Germany). The room temperature pressure dependence of compressibility of the as-prepared metallic glasses was continuously followed using a diamond anvil cell (DAC). The principal diffuse peak was detected in transmission mode to determine the volume changes under high pressure. The energy of the synchrotron radiation was set to $42.85 \mathrm{keV}$, which corresponds to the wavelength of $\lambda=0.28934 \AA$. Kirkpatrick-Baez (KB) mirrors were used to focus photon beam down to $2 \times 2 \mu \mathrm{m}^{2}$. Twodimensional XRD patterns were collected using a fast image plate detector Perkin Elmer 1621 
$\left(2048 \times 2048\right.$ pixels, $200 \times 200 \mu \mathrm{m}^{2}$ pixel size, intensity resolution of 16 bit $)$ carefully mounted orthogonal to the X-ray beam. $\mathrm{CeO}_{2}$ standard from NIST (National Institute of Standards and Technology) was used to calibrate the sample-to-detector distance and tilt of the imaging plate relative to the beam path. Neon was used as a pressure medium loaded in a pressure controller from Sanchez Technology. The pressure acting on the sample was determined using a ruby fluorescence scale from Mao et al. [13]. The pressure was increased in small steps using a pressure membrane attached to the back of the DAC and controlled by a pressure controller from Sanchez Technology. Two-dimensional XRD patterns were integrated to the $q$-space using the software package FIT2D [14].

\section{RESULTS}

\subsection{Pressure dependence of magnetic properties}

Both amorphous Fe-Mn-B alloys with $x=20$ and 24 at $\%$ Mn content studied in this paper show two characteristic magnetic transitions at ambient pressure: (1) a paramagnetic (PM) ferromagnetic (FM) transition at the (ambient-pressure) Curie temperature $T_{\mathrm{C}}=201$ and 159 $\mathrm{K}$ and (2) a spin freezing at temperature $T_{\mathrm{f}}=8.5$ and $13 \mathrm{~K}$, respectively. The Curie point is defined here as the inflection point of the $M(T)$ curves measured in 10 Oe magnetic field. The coherence of this single-field result with the detailed Arrott-plot studies will be described later on. The temperature $T_{\mathrm{C}}$ remarkably decreases with increasing pressure, $\mathrm{d} T_{\mathrm{C}} / \mathrm{d} p=-31 \pm 0.5$ and $-32 \pm 5 \mathrm{~K} / \mathrm{GPa}$ for $x=20$ and 24 at\%, respectively, illustrated in Fig. 1a and Fig. 1b. The low-field magnetization decreases also substantially with increasing pressure and the shape of the curves changes as well. Similar behaviour was also observed for the alloy with $x=24$ at $\%$. 


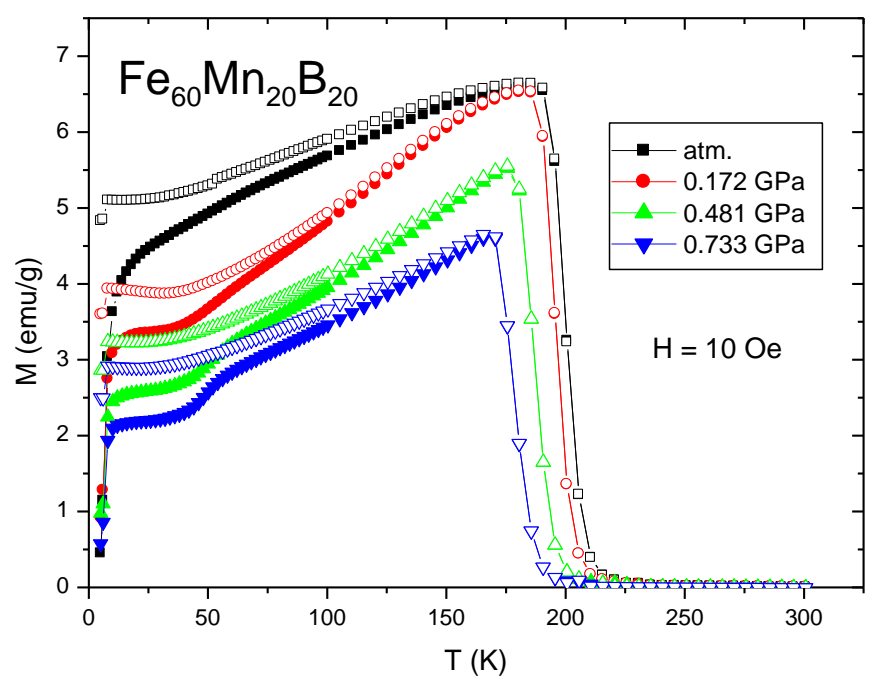

Figure 1a. Temperature dependence of magnetization for $\mathrm{Fe}_{60} \mathrm{Mn}_{20} \mathrm{~B}_{20}$ measured in a magnetic field of 10 Oe after zero-field cooling (ZFC, full symbols) and field cooling in 10 Oe (FC, open symbols) at different pressures indicated.

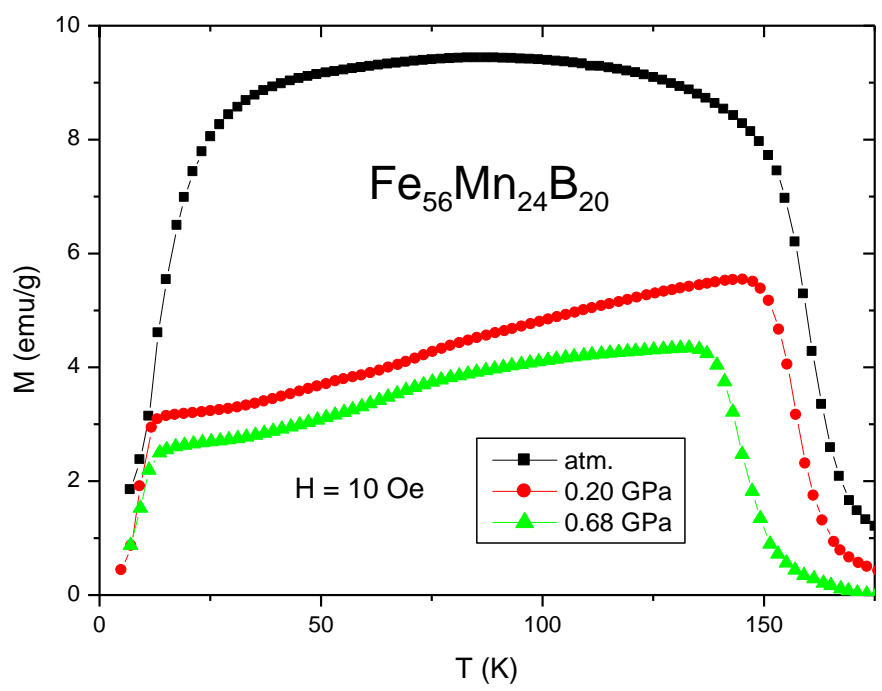

Figure 1b. Temperature dependence of magnetization for 
$\mathrm{Fe}_{56} \mathrm{Mn}_{24} \mathrm{~B}_{20}$ measured in a magnetic field of 10 Oe after zero-field cooling (ZFC, full symbols) and field cooling in 10

Oe (FC, open symbols) at different pressures indicated.

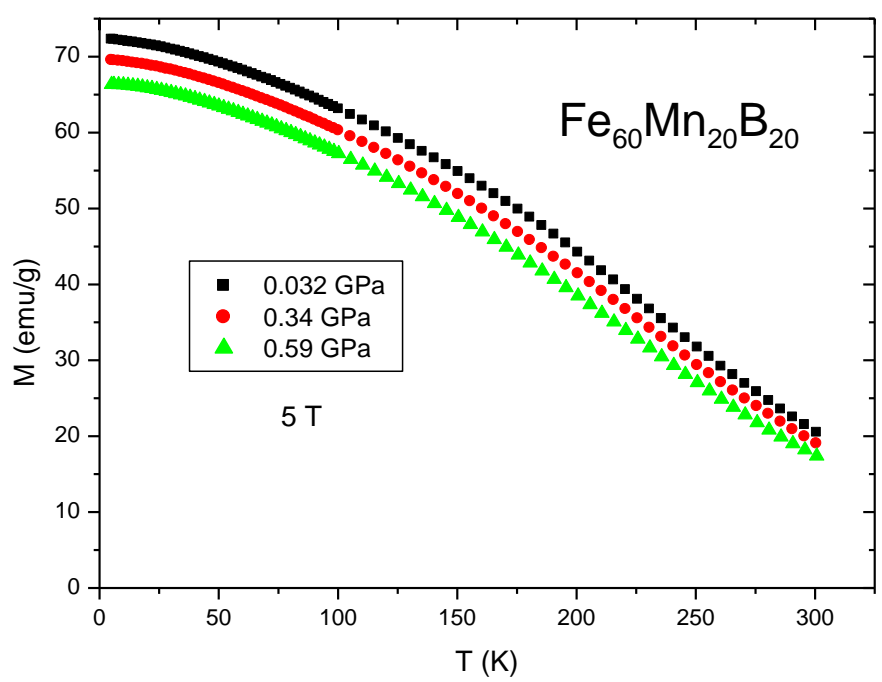

Figure 2. Magnetization measured in $5 \mathrm{~T}$ for $\mathrm{Fe}_{60} \mathrm{Mn}_{20} \mathrm{~B}_{20}$ as a function of temperature at different pressures.

Fig. 2 shows the magnetization measured in a magnetic field of $5 \mathrm{~T}$ for $\mathrm{Fe}_{60} \mathrm{Mn}_{20} \mathrm{~B}_{20}$ as a function of temperature at different pressures. Similar curves were obtained for the alloy with $x=24$ at $\%$. (Note that values of pressure given in Fig. 2 refer to pressure at low temperatures. With increasing temperature, the pressure increases due to the different thermal expansion of the cell and the pressure-transmitting medium (oil). To determine the pressure parameter $\mathrm{d} T_{\mathrm{C}} / \mathrm{d} p$ exactly, the values of pressure at temperatures close to $T_{\mathrm{C}}$ that are given in Fig. 1 were corrected with respect to this effect [12].) The low-temperature saturation magnetization is characterized in two ways: (1) $M_{5 \mathrm{~K}, 5 \mathrm{~T}}$ denotes its value at $5 \mathrm{~K}$ in $5 \mathrm{~T}$ in order to get rid of the errors of extrapolation to $H=0$ and $T=0$; (2) $M_{0}$ is obtained by a linear extrapolation to zero field of high-field portion of the Arrott-plot at $5 \mathrm{~K}$. (Definition and reference to Arrott-plot 
see Section 4.1.) $M_{5 \mathrm{~K}, 5 \mathrm{~T}}$ and $M_{0}$ show a remarkable decrease with increasing pressure, dln $M_{5 \mathrm{~K}, 5 \mathrm{~T}} / \mathrm{d} p=-0.15 \pm 0.02$ and $-0.13 \pm 0.03 \mathrm{GPa}^{-1}, \mathrm{~d} \ln M_{0} / \mathrm{d} p=-0.16 \pm 0.02$ and $-0.15 \pm 0.03$ $\mathrm{GPa}^{-1}$ for $x=20$ and 24 at $\% \mathrm{Mn}$ content, respectively. It is worth noting that the way of definition of the saturation magnetization does not influence its pressure derivative within the experimental error.

As a reference for the effect of the Mn content in the Fe-based glasses on their magnetic properties, we have measured the pressure dependence of the saturation magnetization for the binary $\mathrm{Fe}_{75} \mathrm{~B}_{25}$ amorphous alloy. $M_{5 \mathrm{~K}, 5 \mathrm{~T}}$ and $M_{0}$ show an order of magnitude smaller $\left(\mathrm{d} \ln M_{5 \mathrm{~K}}\right.$, ${ }_{5 \mathrm{~T}} / \mathrm{d} p=-0.016 \pm 0.003 \mathrm{GPa}^{-1}, \mathrm{~d} \ln M_{0} / \mathrm{d} p=-0.012 \pm 0.006 \mathrm{GPa}^{-1}$, respectively) decrease with increasing pressure compared to the ternary Mn-containing alloys.

Figure 3a, Figure $3 \mathrm{~b}$ and Table 1 summarize the pressure effects on both the saturation magnetization and the Curie temperature of the investigated alloys. The decrease of $T_{C}$ under pressure is similar for both Mn-containing alloys; however, taking into account the error bars (Table 1) it does not contradict literature results indicating an increasing trend with decreasing Mn content. ${ }^{5}$ 


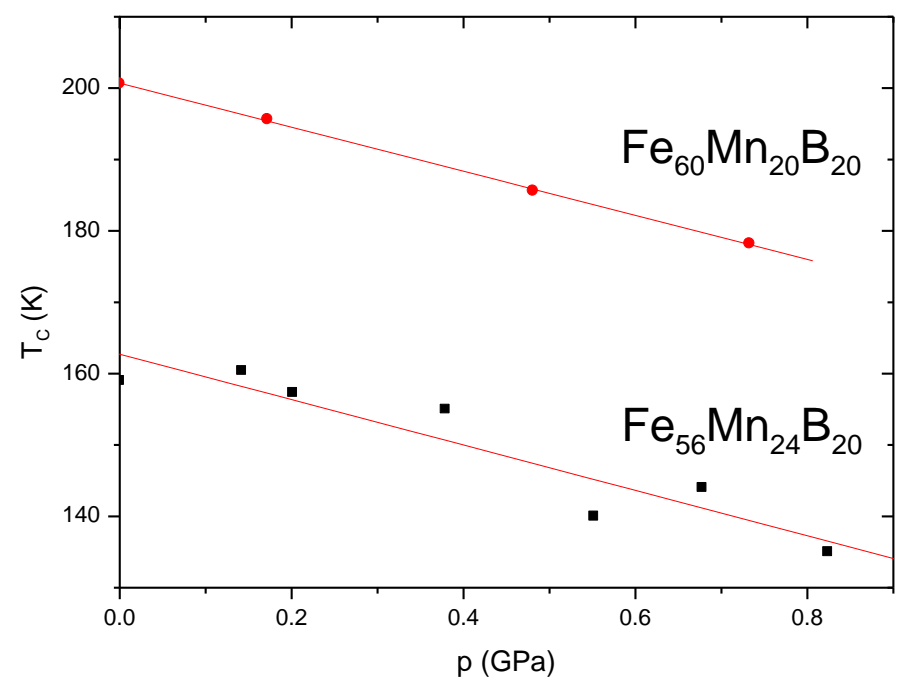

Figure 3a. Pressure effect on Curie temperature for the amorphous $\mathrm{Fe}_{60} \mathrm{Mn}_{20} \mathrm{~B}_{20}$ (circles) and $\mathrm{Fe}_{56} \mathrm{Mn}_{24} \mathrm{~B}_{20}$ (squares) alloys.

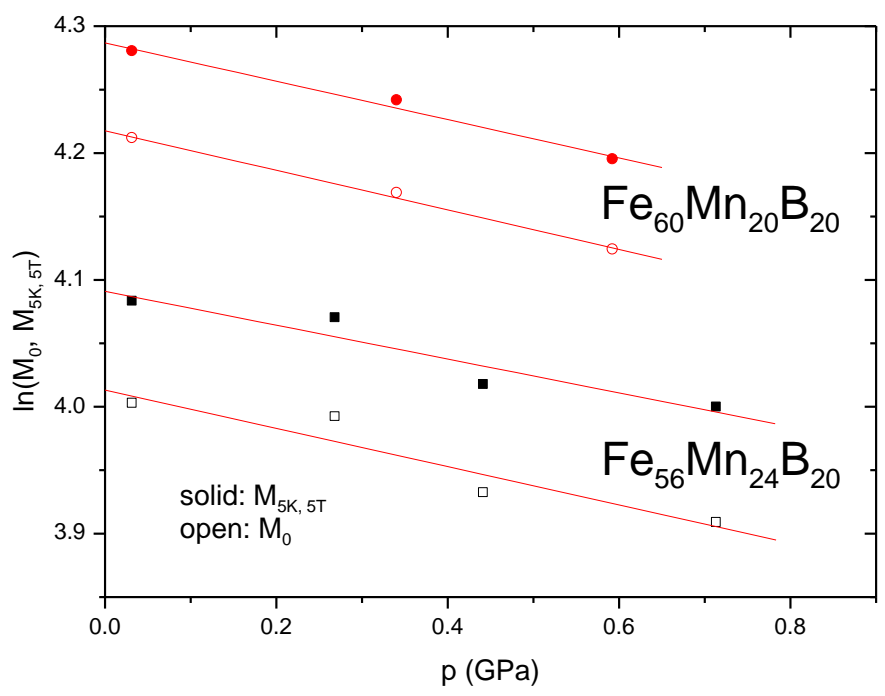

Figure 3b. Pressure effect on saturation magnetization for the amorphous $\mathrm{Fe}_{60} \mathrm{Mn}_{20} \mathrm{~B}_{20}$ (circles) and $\mathrm{Fe}_{56} \mathrm{Mn}_{24} \mathrm{~B}_{20}$ (squares) 
alloys. Solid and open symbols refer to different definitions of the saturation magnetization (see text).

\subsection{Compressibility measurements}

Using in-situ high-pressure X-ray diffraction measurements, we have determined the pressure dependence of the relative volume change $\mathrm{d} V / V_{0}$ for the $\mathrm{Fe}_{56} \mathrm{Mn}_{24} \mathrm{~B}_{20}$ and $\mathrm{Fe}_{75} \mathrm{~B}_{25}$ glasses at ambient temperature up to pressures of $40 \mathrm{GPa}$. It should be noted here that compressibility data presented in Fig. 4 were obtained by tracing the position of the principal diffuse peak [15]. It is worth noting that the ratio between the position of the second and the first diffuse peak does not change with pressure and is almost the same for both alloys, i.e., 1.66. This may suggest that both alloys compress elastically.

Table 1. Pressure dependence of Curie temperature $T_{C}$, saturation magnetization $M_{5 \mathrm{~K}}$, 5T, $M_{0}$ and bulk modulus $B_{0}$.

\begin{tabular}{c|c|c|c}
\hline & $\mathrm{Fe}_{60} \mathrm{Mn}_{20} \mathrm{~B}_{20}$ & $\mathrm{Fe}_{56} \mathrm{Mn}_{24} \mathrm{~B}_{20}$ & $\mathrm{Fe}_{75} \mathrm{~B}_{25}$ \\
\hline$T_{\mathrm{C}}(\mathrm{K})$ & $201 \pm 3^{\mathrm{a}}$ & $159 \pm 3^{\mathrm{a}}$ & $720 \pm 3^{\mathrm{a}, \mathrm{b}}$ \\
\hline $\mathrm{d} T_{\mathrm{C}} / \mathrm{d} p(\mathrm{~K} / \mathrm{GPa})$ & $-31 \pm 0.5$ & $-32 \pm 5$ & $-22.4 \pm 0.2^{\mathrm{b}}$ \\
\hline$M_{5 \mathrm{~K}, 5 \mathrm{~T}}(\mathrm{emu} / \mathrm{g})$ & $70.4 \pm 1^{\mathrm{a}}$ & $59.3 \pm 1^{\mathrm{a}}$ & $187.5 \pm 1$ \\
$M_{0}(\mathrm{emu} / \mathrm{g})$ & $66.0 \pm 1^{\mathrm{a}}$ & $54.7 \pm 1^{\mathrm{a}}$ & $187.1 \pm 1$ \\
\hline$M_{5 \mathrm{~T}, 5 \mathrm{~K}}\left(\mu_{\mathrm{B}} / \mathrm{TM}-\mathrm{at} \%\right)$ & 0.735 & 0.619 & 1.996 \\
\hline $\mathrm{d} \ln M_{5 \mathrm{~T}, 5 \mathrm{~K}} / \mathrm{d} p\left(\mathrm{GPa}^{-1}\right)$ & $-0.15 \pm 0.02$ & $-0.13 \pm 0.03$ & $-0.016 \pm 0.003$ \\
$\mathrm{~d} \ln M_{0} / \mathrm{d} p\left(\mathrm{GPa}^{-1}\right)$ & $-0.16 \pm 0.02$ & $-0.15 \pm 0.03$ & $-0.012 \pm 0.006$ \\
\hline$\kappa\left(10^{-3} \mathrm{GPa}^{-1}\right)$ & - & $6.60 \pm 0.1$ & $5.79 \pm 0.16$
\end{tabular}




\begin{tabular}{c|c|c|c}
$B_{0}(\mathrm{GPa})$ & - & $152 \pm 2$ & $173 \pm 5$ \\
\hline$\Gamma$ & $\sim 22.73$ & 19.70 & 2.76 \\
\hline$a_{2}\left(10^{-5} \mathrm{GPa}^{-2}\right)$ & - & $-6.15 \pm 0.29$ & $-5.13 \pm 0.51$ \\
\hline
\end{tabular}

$T_{\mathrm{C}}$ measured in $10 \mathrm{Oe}$ (see text), $M_{5 \mathrm{~K}, 5 \mathrm{~T}}$ and $M_{0}$ saturation magnetization measured at

5K, 5T and determined from the Arrott-plot, respectively (see text), $\kappa$ compressibility, $B_{0}$ bulk modulus at zero pressure, $\Gamma=\mathrm{d} \ln M_{5 \mathrm{~K}, 5 \mathrm{~T}} / \mathrm{d} \ln V=-(1 / \kappa) * \mathrm{~d} \ln M_{5 \mathrm{~K}, 5 \mathrm{~T}} / \mathrm{d} p$ Grüneisen parameter and $a_{2}$ coefficient of quadratic term in the Bridgman equation of state (see text).

${ }^{\mathrm{a}}$ at $p=1$ bar

${ }^{b}[16]$

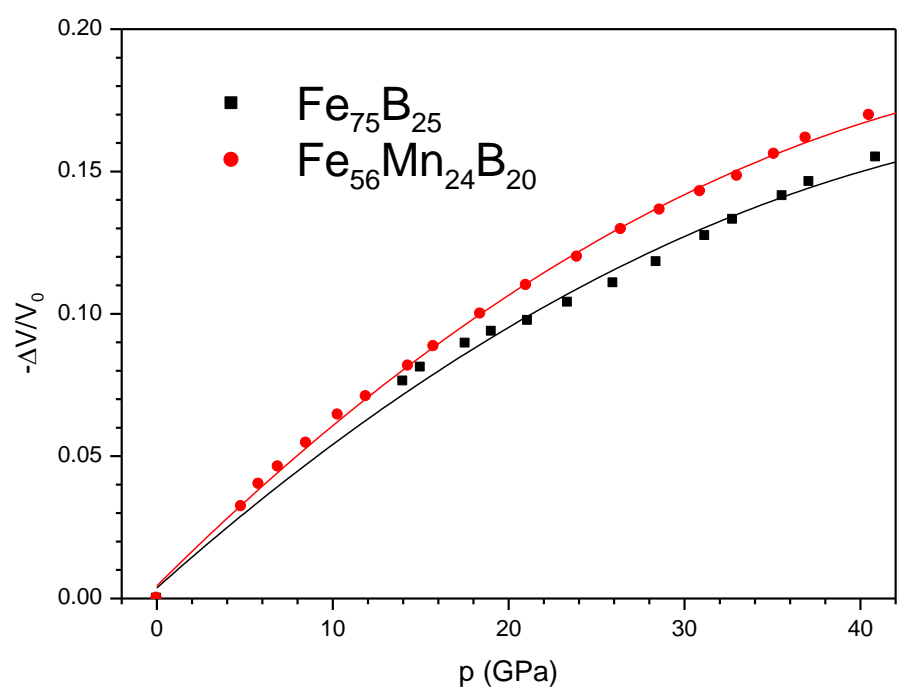

Figure 4. Pressure dependence of relative volume change $\mathrm{d} V / V_{0}$ for $\mathrm{Fe}_{56} \mathrm{Mn}_{24} \mathrm{~B}_{20}$ and $\mathrm{Fe}_{75} \mathrm{~B}_{25}$ glasses at ambient temperature. 
Bridgman [17] has presented the equation of state for compressed solids as follows: $-\Delta V / V_{0}=$ $a_{1} p+a_{2} p^{2}+\ldots$ where $a_{1}(>0)$ and $a_{2}(<0)$ are constants. The bulk modulus at zero pressure $B_{0}=-V_{0}(\mathrm{~d} P / \mathrm{d} V)_{P=0}$ can be calculated as $B_{0}=1 / a_{1}$ where $a_{1}=\kappa$ is called compressibility.

The sample containing Mn exhibits larger volume changes than the binary alloy. The pressure dependence of the relative volume change $\mathrm{d} V / V_{0}$ was fitted to the Bridgman equation of state giving values of bulk moduli for the $\mathrm{Fe}_{56} \mathrm{Mn}_{24} \mathrm{~B}_{20}$ and $\mathrm{Fe}_{75} \mathrm{~B}_{25}$ glasses, which are 152 and 173 GPa, respectively. The values of $\kappa$ and $a_{2}$ from the fit are also shown in Table 1 ; for $\mathrm{Fe}_{60} \mathrm{Mn}_{20} \mathrm{~B}_{20}$ the same value as for $\mathrm{Fe}_{54} \mathrm{Mn}_{24} \mathrm{~B}_{20}$ was used.

\section{DISCUSSION}

\subsection{Arrott-plot analysis: evidence of magnetic heterogeneities}

The magnetic properties of amorphous $\mathrm{Fe}_{80-\mathrm{x}} \mathrm{Mn}_{\mathrm{x}} \mathrm{B}_{20}$ alloys $(0 \leq x \leq 20)$ were studied at ambient pressure with the help of magnetization and Mössbauer measurements by Onodera et al. [4]. Combining these two measurement techniques, they could separate the average magnetic moments carried by the Fe atoms (a decrease from 2.2 to $1.25 \mu_{\mathrm{B}}$ for increasing $x$ was observed) and by the Mn atoms (between 0.5 and $0.85 \mu_{\mathrm{B}}$, with no systematic change beyond the considerable experimental error). Compared to our results, their values of the Curie temperature and saturation magnetization are significantly higher (for the $x=20$ at $\%$ alloy, the Curie point exceeds our value by about $130 \mathrm{~K}$ ). Similar high $T_{\mathrm{C}}$ values were reported by Schiller et al. [5] for the amorphous Fe-Mn-B alloys in the same composition range. The reason for this discrepancy might arise from magnetic heterogeneity of these 
formerly investigated alloys as discussed in detail below. Despite the difference in the absolute value of the Curie temperature, the compositional trend of $T_{\mathrm{C}}$ for our alloys is in line with the literature results, i.e., the higher the Mn content, the lower the Curie point.

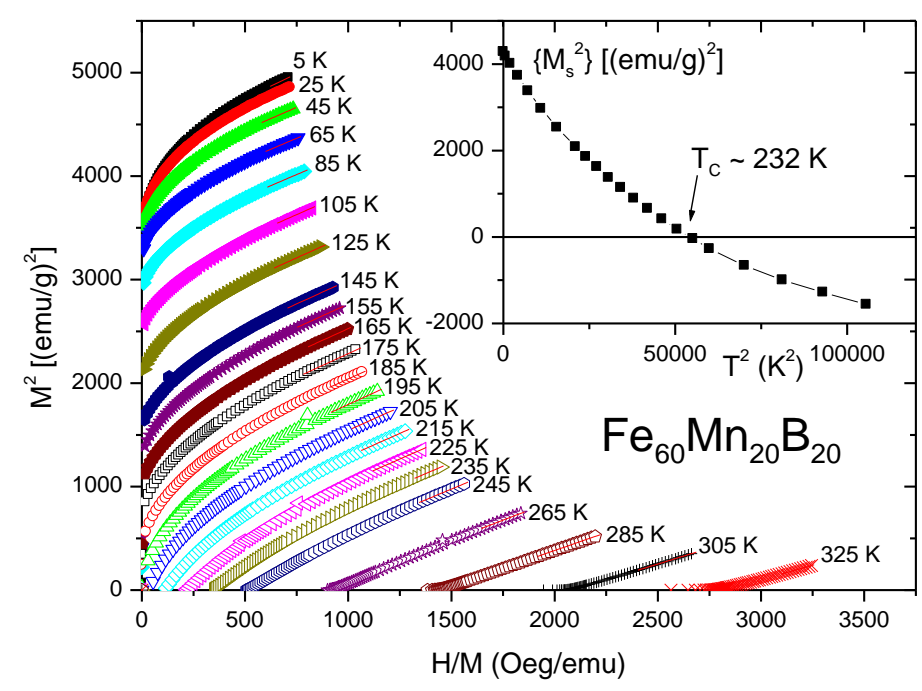

Figure 5. Arrott-plot $\left(M^{2}\right.$ vs. $\left.H / M\right)$ for $\mathrm{Fe}_{60} \mathrm{Mn}_{20} \mathrm{~B}_{20}$ at different temperatures (denoted by label). Inset: $\left\{M_{\mathrm{s}}^{2}\right\}$ vs. $T^{2}$ indicating a high-field Curie temperature of $T_{\mathrm{C}} \sim 232 \mathrm{~K}$ (see text for details).

Figure 5 shows the Arrott-plot $\left(M^{2}\right.$ vs. $\left.H / M\right)$ for $\mathrm{Fe}_{60} \mathrm{Mn}_{20} \mathrm{~B}_{20}$, measured at ambient pressure. It is reasonable to explain the magnetic properties of crystalline and amorphous transition metal alloys (including their pressure dependence) within the framework of the itinerantelectron model using the Stoner theory [1]. For very weak itinerant-electron ferromagnets in the whole temperature range and for strong itinerant-electron ferromagnets around the Curie temperature, the saturation magnetization (extrapolated to $H \rightarrow 0$ in the Arrott-plot) depends on the temperature as $M_{s}{ }^{2} \sim 1-T^{2} / T_{\mathrm{C}}{ }^{2}[18]$ which is shown for this alloy in the inset of Figure 5. (The $\left\{M_{\mathrm{s}}{ }^{2}\right\}$ values plotted in the inset are obtained by a linear extrapolation to $H \rightarrow 0$ of the 
high-field portion of the Arrott-plot (approx. between $3 \mathrm{~T}$ and $5 \mathrm{~T}$ ) at each temperature. Below $T_{\mathrm{C}},\left\{M_{\mathrm{s}}^{2}\right\}=M_{\mathrm{s}}^{2}$ where $M_{\mathrm{s}}$ is the saturation magnetization. Above $T_{\mathrm{C}}$, this construction yields negative values for $\left\{M_{\mathrm{s}}^{2}\right\}$, which has, of course, no physical meaning. In the inset, the negative values of $\left\{M_{\mathrm{s}}{ }^{2}\right\}$ are only used to determine $T_{\mathrm{C}}$ with a better precision.) The visible deviation from linearity around $M_{s}^{2}=0$ (which defines $T_{\mathrm{C}}$ ) hints unambiguously at the presence of magnetic heterogeneity in this amorphous Fe-Mn-B alloy. The high-field value of the Curie temperature is determined to be $T_{\mathrm{C}} \sim 232 \mathrm{~K}$ from the intersection of the extrapolated $M_{s}^{2}$ curve with the $x$-axis. This value is about $30 \mathrm{~K}$ higher than that deduced from the lowfield magnetization curve (Fig. 1, Table 1). The temperature of the isothermal Arrott-curve which goes through the origin is, however, in good agreement with the Curie temperature determined from the single low-field magnetization measurement. Similar results were obtained for $\mathrm{Fe}_{56} \mathrm{Mn}_{24} \mathrm{~B}_{20}$ where the Arrott-plot gives $T_{\mathrm{C}} \sim 196 \mathrm{~K}$. This value is about $35 \mathrm{~K}$ higher than the low-field Curie point (Table 1). The presence of magnetic inhomogeneities in a homogeneous metallic matrix could lead to a substantial "apparent" increase of the Curie temperature when measured in a relatively high field $[19,20]$, i.e., under the conditions of getting the linear sections of the Arrott-plot. Since the degree of the inhomogeneity can vary significantly with the sample preparation conditions and the measured $T_{\mathrm{C}}$ values can depend sensitively on the magnitude of the field applied for the $T_{\mathrm{C}}$ measurement, we think this could be a cause for the scatter of the $T_{\mathrm{C}}$ values found in the literature.

It is worth noting that the increase of the "apparent" Curie temperature with increasing applied magnetic field, determined from the $M(T)$ curves, is a common feature to all Fe-based Invar alloys, either crystalline or amorphous [21]. It is considered to be due to large magnetic fluctuations even up to $T \approx 1.5 T_{\mathrm{C}}$. The magnetic behaviour in these Invar alloys depends 
strongly on the Fe-Fe interatomic distances. Signs of antiferromagnetic interactions are hinted in the vicinity of $2.45 \AA$. In our case we determined the positions of the first coordination shell from the average RDF for $\mathrm{Fe}_{56} \mathrm{Mn}_{24} \mathrm{~B}_{20}$ from the compressibility measurements to vary from 2.53 to $2.40 \AA$ when increasing the pressure from 4 to $40 \mathrm{GPa}$ [22]. This shows that the average Fe-Fe distance does not approach this critical $2.45 \AA$ in the pressure range below 1 GPa investigated by the magnetic (SQUID) measurements.

\subsection{Interpretation of results in the framework of itinerant ferromagnetism}

\subsubsection{Homogeneous vs. inhomogeneous itinerant ferromagnetism}

In order to analyze the observed effect of the hydrostatic pressure on the magnetic properties of amorphous Fe-Mn-B alloys with respect to theoretical model calculations, the volume change of the samples under applied pressures has to be determined. The proportionality factor connecting these quantities is the compressibility $\kappa$. To our knowledge, apart from our measurements presented here, no direct data on compressibility have been available for these alloys. In the literature, a value of $\kappa=7 \times 10^{-3} \mathrm{GPa}^{-1}$ was used for the amorphous $\mathrm{Fe}_{80} \mathrm{~B}_{20}$ alloy [23] while a value of $\kappa=6 \times 10^{-3} \mathrm{GPa}^{-1}$ can be extrapolated from the measured $\kappa$ values of different Fe- and Ni-containing metallic glasses [24,25]. The values of $\kappa=6.60 \times 10^{-3}$ and 5. $79 \times 10^{-3} \mathrm{GPa}^{-1}$ measured for $\mathrm{Fe}_{56} \mathrm{Mn}_{24} \mathrm{~B}_{20}$ and $\mathrm{Fe}_{75} \mathrm{~B}_{25}$, respectively, confirm the estimates presented in the literature.

Based on the Landau-type free energy expansion for a ferromagnet with small homogeneous magnetization, the composition dependence of the pressure derivative of the Curie 
temperature $T_{\mathrm{C}}(x)$ can be described within the itinerant-electron model of ferromagnetism as [26]

$$
\mathrm{d} T_{\mathrm{C}} /\left.\mathrm{d} p\right|_{p=0}=-2 \kappa C \chi_{0} T_{\mathrm{C}}
$$

where $\chi_{0}$ is the ferromagnetic susceptibility at $0 \mathrm{~K}$ and $C$ is the magnetoelastic coupling constant which can be written in the form

$$
C=\frac{5}{12} \frac{I}{I_{b}} \frac{1}{N\left(\varepsilon_{F}\right) \mu_{B}^{2}}-\frac{5}{6} \frac{1}{N\left(\varepsilon_{F}\right) \mu_{B}^{2}} \frac{T_{C}^{2}}{T_{F}^{2}} .
$$

Here $I$ is the effective and $I_{\mathrm{b}}$ the bare (Hartree-Fock) exchange interaction between the itinerant electrons, $N\left(\varepsilon_{\mathrm{F}}\right)$ the density of states at the Fermi energy, $T_{\mathrm{F}}$ the Fermi temperature and $T_{\mathrm{C}}$ the Curie temperature at zero pressure. Substituting for $\chi_{0} \sim T_{\mathrm{C}}^{-2}$ which is valid for homogeneous ferromagnets within the Stoner theory, we obtain [27]

$$
d T_{C} / d p=\frac{5}{3} \kappa T_{C}-\alpha / T_{C}
$$

with $\alpha=\frac{5}{6} \kappa\left(I / I_{b}\right) T_{F}^{2}$. The first term represents the limiting case of strong itinerant ferromagnetism $(\alpha=0)$ and the second term prevails in case of very weak itinerant ferromagnetism ( $\alpha>>\frac{5}{3} \kappa T_{C}^{2}$ ). Similarly, for the temperature dependence of the saturation magnetization we obtain $[16,26]$ 


$$
d \ln M_{0} / d p=d \ln T_{C} / d p-\frac{5}{6} \kappa-\frac{1}{2} d \ln B / d p
$$

where $B$ is the coefficient of the second term $\left(\sim M^{4}\right)$ in the Landau expansion of the free energy. This last term is taken to be small, therefore it can be neglected.

In case of spatial concentration fluctuations present in a ferromagnet, it can be shown [26] that $\chi_{0}$ can be replaced by the spatial average of the susceptibility which does not depend on $T_{\mathrm{C}}$. Hence, eq. (1) gives that $\mathrm{d} T_{\mathrm{C}} / \mathrm{d} p \sim-T_{\mathrm{C}}$ for inhomogeneous ferromagnets. Since the amorphous Fe-Mn-B alloys studied here seem to be magnetically inhomogeneous as discussed above, the results of the pressure dependent measurements should be interpreted within this framework.

\subsubsection{Pressure dependence of the magnetic properties}

of the inhomogenous Fe-Mn-B glassy ferromagnets

The observed decrease of $T_{C}$ of $\mathrm{Fe}_{60} \mathrm{Mn}_{20} \mathrm{~B}_{20}$ under pressure is higher than that reported for an amorphous alloy with the same nominal composition ( $\mathrm{d} T_{\mathrm{C}} / \mathrm{d} p=-22 \mathrm{~K} / \mathrm{GPa}$, [5]). Because of the large scattering of the $T_{\mathrm{C}}$ values and the small $\mathrm{Mn}$-concentration range of the alloys investigated, the composition dependence of the rate of the $T_{\mathrm{C}}$ decrease under pressure cannot be assessed in the present study. However, our results do not contradict the trend described in the literature, i.e., the higher the Mn content in the alloy, the lower $T_{\mathrm{C}}$ and the lower the rate of the pressure decrease of $T_{\mathrm{C}}$. This tendency follows a general trend observed for amorphous alloys, i.e., the lower the Curie temperature, the smaller the absolute value of the pressure 
derivative [28]. This trend corresponds to the pressure dependence of the Curie point expected in inhomogeneous ferromagnets discussed above. The same trend is true for high $\mathrm{Cr}$-content amorphous Fe-Cr-B alloys [29]. As mentioned above, even the smaller values of $\mathrm{d} T_{\mathrm{C}} / \mathrm{d} p$ measured by Schiller et al. [5] are in accordance with the expected $\mathrm{d} T_{\mathrm{C}} / \mathrm{d} p \sim-T_{\mathrm{C}}$ trend together with our ones. The opposite trend is observed for Fe-based crystalline alloys containing magnetic late TM atoms of different types and for metallic glasses considered to be homogeneous (e.g. Fe-rich binary amorphous alloys like Fe-B, low $\mathrm{Cr}$-content Fe-Cr-B glasses) [16]. A common feature of these crystalline and amorphous materials is the Invar behaviour.

As an alternative to the inhomogeneous model, the cation mixing model $[5,28]$ is frequently used to explain the $\mathrm{d} T_{\mathrm{C}} / \mathrm{d} p \sim-T_{\mathrm{C}}$ dependence observed in some TM-containing amorphous alloys within the framework of a homogeneous model. The main idea is the existence of competing contributions to the pressure derivatives of $T_{\mathrm{C}}$, originating from different atomic pairs. However, the quantitative description is problematic and the framework used is far from our modern understanding of metallic magnetism, where the Curie point is determined by long range interactions.

A further direct evidence of the itinerancy of these Fe-Mn-B metallic glasses is a rapid decrease of the magnetization per transition metal atom (TM-at.) with the increase of the Mn content as shown in Table 1. This is well comparable with the same effect in Fe-Cr-B glasses [29]. It is evident from eq. (3) that the pressure derivatives of $T_{\mathrm{C}}$ and the saturation magnetization $M_{5 \mathrm{~K}, 5 \mathrm{~T}}$ differ such that $\mathrm{d} \ln M_{5 \mathrm{~K}, 5 \mathrm{~T}} / \mathrm{d} p<\mathrm{d} \ln T_{\mathrm{C}} / \mathrm{d} p$ since the compressibility $\kappa$ is always positive. In our case, for eq. (3) we obtain $-0.15 \approx-0.154-0.0055\left(\mathrm{Fe}_{60} \mathrm{Mn}_{20} \mathrm{~B}_{20}\right)$, - 
$0.13 \approx-0.20-0.0055\left(\mathrm{Fe}_{56} \mathrm{Mn}_{24} \mathrm{~B}_{20}\right)$ and $-0.016>-0.031-0.0048\left(\mathrm{Fe}_{75} \mathrm{~B}_{25}\right)$, where all data are in $\mathrm{GPa}^{-1}$. The good agreement of the experimental data with eq. (3) for the Mn-containing glasses shows a pronounced itinerancy of the amorphous Fe-Mn-B alloys. The lower sensitivity of magnetization of the $\mathrm{Fe}_{75} \mathrm{~B}_{25}$ glass to external pressure at temperature $5 \mathrm{~K}$ characterizes this glass as a weak itinerant ferromagnet for which eq. (3) is valid at around $T_{\mathrm{C}}$ only.

Theoretical calculations of the relation between the local magnetic Fe moment at $0 \mathrm{~K}$ and the local volume in amorphous iron [30] and in amorphous Fe-B alloys [31] were presented. The latter work predicts $0.3 \%$ change of the average Fe moment upon $0.1 \%$ change of the atomic volume. This corresponds to $\Gamma=3$ for the dimensionless Grüneisen parameter where $\Gamma=$ $\mathrm{d} \ln M_{5 \mathrm{~K}, 5 \mathrm{~T}} / \mathrm{d} \ln V=-(1 / \kappa)^{*} \mathrm{~d} \ln M_{5 \mathrm{~K}, 5 \mathrm{~T}} / \mathrm{d} p$. Our measured value for amorphous $\mathrm{Fe}_{75} \mathrm{~B}_{25}$ is $\Gamma=2.76$ (Table 1) which is rather close to the theoretical prediction. In our study, the Grüneisen parameter amounts to $\Gamma=22.73$ and 19.70 for $x=20$ and 24 at $\%$ Mn content, respectively (Table 1). These values are approx. ten times higher than that measured for the binary $\mathrm{Fe}_{75} \mathrm{~B}_{25}$ amorphous alloy (Table 1) and obtained from theoretical calculations for binary Fe-B alloys, indicating a significant influence of $\mathrm{Mn}$ on the volume instability of the magnetic moment. The high-pressure Mössbauer spectroscopy measurements on amorphous $\mathrm{Fe}_{3} \mathrm{~B}$ [8] at $300 \mathrm{~K}$ also showed a relatively small decrease of the average hyperfine field, $B_{\mathrm{hf}}\left(\mathrm{d} \ln B_{\mathrm{hf}} / \mathrm{d} p=-\right.$ $\left.0.0089 \mathrm{GPa}^{-1}\right)$.

A relatively small decrease of the saturation magnetization under high pressure up to $0.4 \mathrm{GPa}$ $\left(\mathrm{d} \ln M_{0} / \mathrm{d} p=-0.02 \pm 0.005 \mathrm{GPa}^{-1}\right)$ was found for the $\mathrm{Fe}_{40} \mathrm{Ni}_{40} \mathrm{P}_{14} \mathrm{~B}_{6}$ metallic glass at room 
temperature [6]. The Curie point of this alloy is around $T_{\mathrm{C}}=473 \mathrm{~K}\left(200^{\circ} \mathrm{C}\right)$, therefore the relevance of this room-temperature measurement may be questioned. In any case, this value of $\mathrm{d} \ln M_{0} / \mathrm{d} p$ is close to that measured for the binary $\mathrm{Fe}_{75} \mathrm{~B}_{25}$ amorphous alloy (Table 1). The pressure-induced relative hyperfine-field change in the amorphous $\left(\mathrm{Fe}_{0.25} \mathrm{Ni}_{0.75}\right)_{3} \mathrm{~B}$ alloy at 4.2 $\mathrm{K}\left(-0.01 \mathrm{GPa}^{-1}[9]\right)$ is smaller than the relative magnetization decrease observed in the above Ni rich amorphous alloys; this fact might however be connected with the rather different composition. It is also smaller than the pressure-induced relative saturation magnetization change for the binary $\mathrm{Fe}_{75} \mathrm{~B}_{25}$ amorphous alloy (Table 1).

Since the pressure dependence of the hyperfine field (approximately proportional to the Fe magnetic moment) seems to be weak in binary amorphous alloys, the much more pronounced pressure dependence of the saturation magnetization in Fe-Mn-B might be associated with the steeper decrease of the magnetic moment of Mn atoms under pressure. In case of our $\mathrm{Fe}_{60} \mathrm{Mn}_{20} \mathrm{~B}_{20}$ amorphous alloy, assuming no change of the Fe magnetic moment under the maximum applied pressure of $0.6 \mathrm{GPa}$, a decrease of the Mn magnetic moment from 0.85 to 0.6-0.7 $\mu_{\mathrm{B}}$ would explain the total $5.7 \%$ change of the saturation magnetization measured at this pressure. It is an open and interesting question whether with a further pressure increase, the $\mathrm{Mn}$ moment remains zero or could become negative (antiferromagnetically aligned Mn moments) in this type of ternary amorphous alloys.

A trend seems to emerge from these observations in that the pressure derivative of the saturation magnetization is much greater in case of amorphous alloys containing early transition-metal atoms ( $\mathrm{Mn}$ in the present work) besides iron. A similar tendency might also 
be inferred from the more significant pressure dependence of the Fe hyperfine field $\left(H_{\mathrm{eff}}\right)$ in a Cr-containing alloy $\left(\mathrm{d} \ln H_{\text {eff }}(T) / \mathrm{d} p=-0.10 \mathrm{GPa}^{-1}\right.$ for $\left.\mathrm{Fe}_{32} \mathrm{Ni}_{36} \mathrm{Cr}_{14} \mathrm{P}_{12} \mathrm{~B}_{6}\right)$ with respect to that of a Cr-free alloy $\left(\mathrm{d} \ln H_{\text {eff }}(T) / \mathrm{d} p=-0.029 \mathrm{GPa}^{-1}\right.$ for $\left.\mathrm{Fe}_{27} \mathrm{Ni}_{53} \mathrm{P}_{14} \mathrm{~B}_{6}\right)$ [7]. However, for the lack of enough data, it is not yet clear if this feature is related to the "early"-type of the TM elements or it is associated with antiferromagnetic interactions observed in alloys with $\mathrm{Mn}$ and $\mathrm{Cr}$. Hydrostatic pressure also reduces TM-Fe distances and causes a further reduction of Fe moment due to an enhanced magnetic-moment perturbation on account of d-p electron hybridization.

Finally, it is stimulating to compare our results with measurements [32,33] made under tensile stress ("negative pressure"). Qualitatively our results are in line with these studies. The sign of the $T_{\mathrm{C}}$ change is opposite as it is expected, the values reported in these papers $(3,14$ and 19 $\mathrm{K} \mathrm{GPa}^{-1}$ for $\mathrm{Fe}_{80} \mathrm{Zr}_{10} \mathrm{~B}_{10}, \mathrm{Fe}_{87} \mathrm{Zr}_{6} \mathrm{Cu}_{1} \mathrm{~B}_{6}$ and $\left(\mathrm{Fe}_{0.92} \mathrm{Zr}_{0.08}\right)_{96} \mathrm{~B}_{4}$, respectively) are in the same order of magnitude but slightly smaller than our values of about $30 \mathrm{~K} \mathrm{GPa}^{-1}$. Since the compositions are completely different from ours, the deviations can be understood.

\section{CONCLUSIONS}

A relatively large pressure effect on the Curie temperature and the saturation magnetization was observed for amorphous $\mathrm{Fe}_{60} \mathrm{Mn}_{20} \mathrm{~B}_{20}$ and $\mathrm{Fe}_{56} \mathrm{Mn}_{24} \mathrm{~B}_{20}$ alloys compared to binary amorphous $\mathrm{Fe}_{75} \mathrm{~B}_{25}$. Similarly, the decrease of the saturation magnetization is also one order of magnitude larger than that expected from reported theoretical calculations for amorphous iron and amorphous Fe-B alloys. The results were interpreted within an inhomogeneous itinerant-electron model of ferromagnetism. Theoretical ab-initio calculations are, however, 
necessary to properly assess the validity of currently available models of magnetism for the Fe-based amorphous and/or nanostructured alloys.

\section{ACKNOWLEDGMENTS}

This work was supported by the Hungarian Scientific Research Fund under Grant OTKA K 101456. The fruitful discussion with Prof. I. Vincze and Dr. I. Bakonyi is highly acknowledged. We thank L. Bujdosó for the sample preparation and Dr. L. Pogány for the microprobe analysis. Financial support from Zhejiang University-Helmholtz cooperation fund is gratefully acknowledged. The use of the Infrastructure for Extreme Condition Research (ECSI) at PETRA III is acknowledged for gas loading samples and ruby fluorescence measurements. 


\section{References}

[1] Wohlfarth E P 1983 in: Amorphous Metallic Alloys ed Luborsky F E (London: Butterworth) p 283

[2] Nielsen H. J. V 1979 J Magn. Magn. Mater. 12187

[3] Nielsen H J V 1979 Solid State Commun. 30239

[4] Onodera H and Yamamoto H 1981 J. Phys. Soc. Japan 503575

[5] Schiller J and Wisniewski R 1989 J. Magn. Magn. Mater. 80318

[6] Anderson III P M and Lord E A Jr 1977 J. Appl. Phys. 484839

[7] Bouzabata B, Ingalls R and Rao K V 1984 Phys. Rev. B 296105

[8] Abd-Elmeguid M M, Micklitz H and Vincze I 1982 Phys. Rev. B 251

[9] Eickelmann H J, Abd-Elmeguid M M, Micklitz H and Brand R A 1984 Phys. Rev. B 29 2443

[10] van der Woude F and Vincze I 1980 J. de Physique 41 C1-151

[11] Dubiel S M 2010 J. Alloys and Compounds 48818

[12] Kamarád J, Machátová Z and Arnold Z 2004 Rev. Sci. Instrum. 755022

[13] Mao H K, Xu J and Bell P M 1986 J. Geophys. Res. 914673

[14] Hammersley A P, Svensson S O, Hanfland M, Fitch A N and Häusermann D 1996 High Press. Res. 14235

[15] Wang J F, Li R, Xiao R J, Xu T, Li Y, Liu Z Q, Huang L, Hua N, Li G, Li Y C and Zhan T 2011 Appl. Phys. Lett. 99151911

[16] Kamarád J and Arnold Z 1986 Physica B 139-140 382

[17] Bridgman P W 1958 The Physics of High Pressure (London: Bell and Sons)

[18] Mathon J 1968 Proc. Roy. Soc. A 306355 
[19] Acker F and Huguenin R 1979 J. Magn. Magn. Mater. 1258

[20] Bakonyi I, Burgstaller A, Socher W, Voitländer J, Tóth-Kádár E, Lovas A, Ebert H, Wachtel E, Willman N and Liebermann H H 1993 Phys. Rev. B 4714961

[21] Fdez-Gubieda M L, García-Arribas A, Barandiarán J M, López Antón R, Orue I, Gorria P, Pizzini S and Fontaine A 2000 Phys. Rev. B 625746

[22] Bednarcik J, Kiss L F, Konôpková Z and Liermann H P 2011 Compressibility studies of Fe-B metallic glasses, HASYLAB Annual Report

[23] Fukamichi K 1983 in: Amorphous Metallic Alloys ed Luborsky F E (London: Butterworth) p 317

[24] Chou C-P, Davis L A and Narasimhan M C 1977 Scr. Metall. 11417

[25] Chou C-P, Davis L A and Hasegawa R 1979 J. Appl. Phys. 503334

[26] Wagner D and Wohlfarth E P 1981 J. Phys. F: Metal Phys. 112417

[27] Edwards D M and Wohlfarth E P 1968 Proc. Roy. Soc. A 303127

[28] Medvedeva I V, Bersenev Yu S, Ganin A A, Bärner K, Schünemann J-W and Heinemann K 1993 J. Magn. Magn. Mater. 124293

[29] Kamarád J, Arnold Z and Nielsen H J V 1981 J. Magn. Magn. Mater. 2369

[30] Krauss U and Krey U 1991 J. Magn. Magn. Mater. 98 L1

[31] Hafner J, Tegze M and Becker Ch 1994 Phys. Rev. B 49285

[32 ] Barandiarán J M, Gorria P, Orúe I, Fdez-Gubieda M L, Plazaola F and Hernando A 1996 Phys. Rev. B 543026

[33] Gondro J, Świerczek J, Rjącki J, Ciurzyńska W, Olszewski J, Zbroszczyk J, Błoch K, Osyra M and Łukiewska A 2013 J. Magn. Magn. Mater. 341100 
Table 1. Pressure dependence of Curie temperature $T_{C}$, saturation magnetization $M_{5 \mathrm{~K}}$, 5T, $M_{0}$ and bulk modulus $B_{0}$.

\begin{tabular}{c|c|c|c}
\hline & $\mathrm{Fe}_{60} \mathrm{Mn}_{20} \mathrm{~B}_{20}$ & $\mathrm{Fe}_{56} \mathrm{Mn}_{24} \mathrm{~B}_{20}$ & $\mathrm{Fe}_{75} \mathrm{~B}_{25}$ \\
\hline$T_{\mathrm{C}}(\mathrm{K})$ & $201 \pm 3^{\mathrm{a}}$ & $159 \pm 3^{\mathrm{a}}$ & $720 \pm 3^{\mathrm{a}, \mathrm{b}}$ \\
\hline $\mathrm{d} T_{\mathrm{C}} / \mathrm{d} p(\mathrm{~K} / \mathrm{GPa})$ & $-31 \pm 0.5$ & $-32 \pm 5$ & $-22.4 \pm 0.2^{\mathrm{b}}$ \\
\hline$M_{5 \mathrm{~K}, 5 \mathrm{~T}}(\mathrm{emu} / \mathrm{g})$ & $70.4 \pm 1^{\mathrm{a}}$ & $59.3 \pm 1^{\mathrm{a}}$ & $187.5 \pm 1$ \\
$M_{0}(\mathrm{emu} / \mathrm{g})$ & $66.0 \pm 1^{\mathrm{a}}$ & $54.7 \pm 1^{\mathrm{a}}$ & $187.1 \pm 1$ \\
\hline$M_{5 \mathrm{~T}, 5 \mathrm{~K}}\left(\mu_{\mathrm{B}} / \mathrm{TM}-\mathrm{at} \%\right)$ & 0.735 & 0.619 & 1.996 \\
\hline $\mathrm{d} \ln M_{5 \mathrm{~T}, 5 \mathrm{~K}} / \mathrm{d} p\left(\mathrm{GPa}^{-1}\right)$ & $-0.15 \pm 0.02$ & $-0.13 \pm 0.03$ & $-0.016 \pm 0.003$ \\
$\mathrm{~d} \ln M_{0} / \mathrm{d} p\left(\mathrm{GPa}{ }^{-1}\right)$ & $-0.16 \pm 0.02$ & $-0.15 \pm 0.03$ & $-0.012 \pm 0.006$ \\
\hline$\kappa\left(10^{-3} \mathrm{GPa}^{-1}\right)$ & - & $6.60 \pm 0.1$ & $5.79 \pm 0.16$ \\
\hline$B_{0}(\mathrm{GPa})$ & - & $152 \pm 2$ & $173 \pm 5$ \\
\hline$\Gamma$ & $\sim 22.73$ & 19.70 & 2.76 \\
\hline$a_{2}\left(10^{-5} \mathrm{GPa}^{-2}\right)$ & - & $-6.15 \pm 0.29$ & $-5.13 \pm 0.51$ \\
\hline$T_{\mathrm{C}} \mathrm{m}$ & & & \\
\hline
\end{tabular}

$T_{\mathrm{C}}$ measured in $10 \mathrm{Oe}$ (see text), $M_{5 \mathrm{~K}, 5 \mathrm{~T}}$ and $M_{0}$ saturation magnetization measured at $5 \mathrm{~K}, 5 \mathrm{~T}$ and determined from the Arrott-plot, respectively (see text), $\kappa$ compressibility, $B_{0}$ bulk modulus at zero pressure, $\Gamma=\mathrm{d} \ln M_{5 \mathrm{~K}, 5 \mathrm{~T}} / \mathrm{d} \ln V=-(1 / \kappa) * \mathrm{~d} \ln M_{5 \mathrm{~K}, 5 \mathrm{~T}} / \mathrm{d} p$ Grüneisen parameter and $a_{2}$ coefficient of quadratic term in the Bridgman equation of state (see text).

at $p=1$ bar

${ }^{b}[16]$ 


\section{Figure Captions}

Fig. 1a. Temperature dependence of magnetization for $\mathrm{Fe}_{60} \mathrm{Mn}_{20} \mathrm{~B}_{20}$ measured in a magnetic field of 10 Oe after zero-field cooling (ZFC, full symbols) and field cooling in 10 Oe (FC, open symbols) at different pressures indicated.

Fig. 1b. Temperature dependence of magnetization for $\mathrm{Fe}_{56} \mathrm{Mn}_{24} \mathrm{~B}_{20}$ measured in a magnetic field of 10 Oe after zero-field cooling (ZFC, full symbols) and field cooling in $10 \mathrm{Oe}$ (FC, open symbols) at different pressures indicated.

Fig. 2. Magnetization measured in $5 \mathrm{~T}$ for $\mathrm{Fe}_{60} \mathrm{Mn}_{20} \mathrm{~B}_{20}$ as a function of temperature at different pressures.

Fig. 3a. Pressure effect on Curie temperature for the amorphous $\mathrm{Fe}_{60} \mathrm{Mn}_{20} \mathrm{~B}_{20}$ (circles) and $\mathrm{Fe}_{56} \mathrm{Mn}_{24} \mathrm{~B}_{20}$ (squares) alloys.

Fig. 3b. Pressure effect on saturation magnetization for the amorphous $\mathrm{Fe}_{60} \mathrm{Mn}_{20} \mathrm{~B}_{20}$ (circles) and $\mathrm{Fe}_{56} \mathrm{Mn}_{24} \mathrm{~B}_{20}$ (squares) alloys. Solid and open symbols refer to different definitions of the saturation magnetization (see text).

Fig. 4. Pressure dependence of relative volume change $d V / V_{0}$ for $\mathrm{Fe}_{56} \mathrm{Mn}_{24} \mathrm{~B}_{20}$ and $\mathrm{Fe}_{75} \mathrm{~B}_{25}$ glasses at ambient temperature. 
Fig. 5. Arrott-plot $\left(M^{2}\right.$ vs. $\left.H / M\right)$ for $\mathrm{Fe}_{60} \mathrm{Mn}_{20} \mathrm{~B}_{20}$ at different temperatures (denoted by label). Inset: $M_{\mathrm{S}}^{2}$ vs. $T^{2}$ indicating a high-field Curie temperature of $T_{\mathrm{C}} \sim 232 \mathrm{~K}$ (see text for details). 